\title{
An outbreak of a possibly new Salmonella enterica subspecies enterica serovar with the antigenic formula 11:z41:e,n,z15, Greece, March to May 2016: preliminary results
}

G Mandilara ${ }^{1}$, K Mellou ${ }^{2}$, K Karadimas ${ }^{1}$, L Georgalis ${ }^{3}$, M Polemis ${ }^{4}$, T Georgakopoulou 2 , A Vatopoulos ${ }^{1}$

1. National Reference Centre for Salmonella and Shigella, National School of Public Health \& Central Public Health Laboratory, Hellenic Centre of Disease Control and Prevention, Vari, Greece

2. Hellenic Centre of Disease Control and Prevention, Athens, Greece

3. European Program for Intervention Epidemiology Training (EPIET), European Centre for Disease Prevention and Control, (ECDC), Stockholm, Sweden

4. Central Public Health Laboratory, Hellenic Centre of Disease Control and Prevention, Vari, Greece

Correspondence: Georgia Mandilara (gmandilara@esdy.edu.gr)

Citation style for this article:

Mandilara G, Mellou K, Karadimas K, Georgalis L, Polemis M, Georgakopoulou T, Vatopoulos A. An outbreak of a possibly new Salmonella enterica subspecies enterica serovar with the antigenic formula 11:z41:e,n,z15, Greece, March to May 2016: preliminary results. Euro Surveill. 2016;21(25):pii=30265. D0I: http:// dx.doi.org/10.2807/1560-7917.ES.2016.21.25.30265

Article submitted on 03 June 2016 / accepted on 23 June 2016 / published on 23 June 2016

Eleven Salmonella spp. isolates with the antigenic type 11:z41:e,n,z15 - not referred to in the gth edition of the White-Kauffman-Le Minor Scheme - were identified at the National Reference Laboratory for Salmonella in Greece. Their pulsed-field gel electrophoresis profiles were indistinguishable. No apparent epidemiological link has yet been identified; the results of a case-case study are pending.

Between 24 March and 27 May 2016, eleven Salmonella spp. isolates with an unusual antigenic type were identified by the National Reference Laboratory for Salmonella and Shigella (NRLSS) in Greece.

The antigenic type of the eleven isolates was $11: z_{41}: e, n, z_{15}$, which is not referred to in the gth edition of the White-Kauffman-Le Minor Scheme [1]. The isolates were cultured from stool and collected from ten patients with diarrhoea; for one asymptomatic case, the sample was obtained during a routine test for acquiring a certificate for occupational use. However, the latter case reported having had gastroenteritis symptoms some weeks before. None of the isolates fermented malonate but all fermented dulcitol, indicating that they belong to Salmonella enterica subspsecies enterica [1]. All were susceptible to the laboratory routine panel of antimicrobial agents, including third generation cephalosporins and fluoroquinolones. Tests were performed using the disk diffusion method and breakpoints according to the European Committee on Antimicrobial Susceptibility Testing (EUCAST) were applied [2].The pulsed-field gel electrophoresis (PFGE) profiles, after digestion with $\mathrm{Xbal}$ according to
PulseNet protocol [3], were indistinguishable in 10 of the 11 strains (one result of the last isolated strain is pending) (Figure 1).

According to the database of the NRLSS and of the Veterinary Reference Laboratory for Greece, the antigenic type $11: z_{41}: e, n, z_{15}$ has never been identified before from animals, animal products or food samples.

\section{Description of cases and epidemiological curve}

All cases, defined as persons with diarrhoea with the new Salmonella enterica subsp. enterica serovar $(n=10)$, were of Greek nationality (6 male, 4 female). Eight of the cases were children (15 months-3 years old) and two were adults (28-6o years of age). Cases' symptoms included diarrhoea (one case had bloody diarrhoea), vomiting and abdominal cramps, and three of them reported fever $\left(\geq 38.0^{\circ} \mathrm{C}\right)$. Three cases reported relapse of symptoms and all cases will be followed up. Three of the identified cases reported a household contact with similar symptoms (another child in the same family). Investigation of the possible household clusters showed that they were most probably co-infected but none of the household contacts was laboratory-confirmed. In this report, only information for laboratory-confirmed cases is presented.

The majority of the cases $(n=9)$ were scattered in the region of Attica, one case was identified in Kastoria in northern Greece, and another in Korinthos, central Greece. Only one case reported travel abroad to Torino, in Italy, five days before the symptom onset. This 
PFGE profile of Salmonella enterica subsp. enterica isolates with antigenic formula $11: \mathrm{z}_{41}: \mathrm{e}, \mathrm{n}, \mathrm{z}_{15}$, Greece, April to May 2016 $(\mathrm{n}=10)$

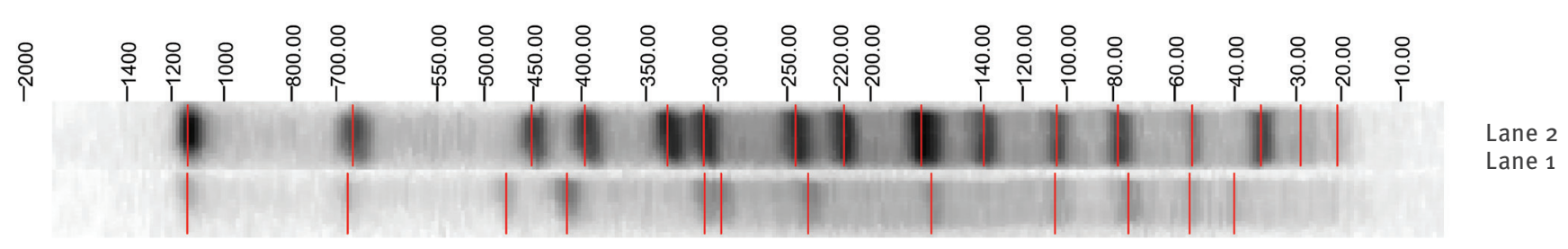

PFGE: pulsed-field gel electrophoresis.

Lane 1: new serovar profile.

Lane 2: Salmonella serotype Braenderup H9812 universal size standard.

PGFE profiles from all cases were indistinguishable.

case stated developing symptoms before returning to Greece. Figure 2 presents the temporal distribution of cases by week of symptom onset.

\section{Actions taken}

Communication of findings to other countries The PFGE profile [4] was uploaded to the European Surveillance System (TESSy) operated by the European Centre for Disease Prevention and Control (ECDC) (ECDC_ID: f5fo517b-f809-4d2b-973f-3f9c52ob9d77) and an urgent inquiry (UI) was launched via the ECDC's Epidemic Intelligence Information System (EPIS) (UI358). According to the ECDC food- and waterborne diseases curators, no isolates with a matching PFGE profile (Xbal.2460) have been reported to TESSy (personal communication, Saara M. Kotila, ECDC, 25 May 2016). Moreover, none of the 15 countries that replied to the UI had identified the new serovar in the past.

Three of the isolates were sent to the World Health Organization (WHO) Collaborating Centre for Reference and Research on Salmonella at Pasteur Institute in Paris, France, which is responsible for the validation of new serovars. According to Pasteur Institute, the isolates represent a putative new serotype of Salmonella enterica subsp. enterica (personal communication, Francois-Xavier Weill, Pasteur Institute, 26 May 2016).

\section{Investigation of cases}

Laboratory confirmed cases were interviewed by telephone with a standard trawling questionnaire for investigating salmonellosis cases but no apparent epidemiological link has yet been identified. Cases were geographically scattered, had not travelled inside the country, did not have pets or contact with reptiles, and had not participated in any common activities. Based on the results from the trawling questionnaires, no food item emerged as possible source of the infections.
Thus, it was decided to further investigate this salmonellosis cluster by performing an analytical study. Given the highly selective nature of food-borne case reporting and in order to reduce recall bias, a casecase study for the identification of possible risk factors was designed [5-7]. This study included a comparison group of Salmonella Enteritidis cases from the Greek Mandatory Notification System (MNS) matched by age ( \pm 1 year), and place of residence. In order to increase the power of the study, the ratio of case-case 1:3 was decided.

A structured web-based trawling questionnaire, containing a long list of possible exposures (food and water consumption, exposures to animals, travel history, activities, etc.) was developed and distributed to all cases (both of unknown Salmonella serovar and SalmonellaEnteritidis).

\section{Conclusions}

According to some preliminary findings, a new Salmonella enterica sub. enterica serovar seems to have caused an outbreak in Greece over two months in the first half of 2016, with 10 cases (and one asymptomatic) as of 27 May. Reported cases are mostly children, however this may be influenced by the fact that laboratory tests are performed more frequently in children with gastroenteritis symptoms than in adults with the same symptoms. We cannot be sure about the geographical distribution of cases. The higher number of cases from Attica may be because more isolates are sent to the National Reference Laboratory from this region. Three cases reported relapse of symptoms. Data on the severity of the disease are also gathered and a case-case study is underway. Final results are pending.

We encourage other countries to contact authors in case of identifying isolates of the new serovar of 


\section{FIGURE 2}

Distribution of symptomatic Salmonella enteric subsp. enterica cases of a possibly new serovar with the antigenic formula $11: z_{41}:$ enz $_{15}$, by week of symptom onset, Greece, April to May $2016(\mathrm{n}=10)$

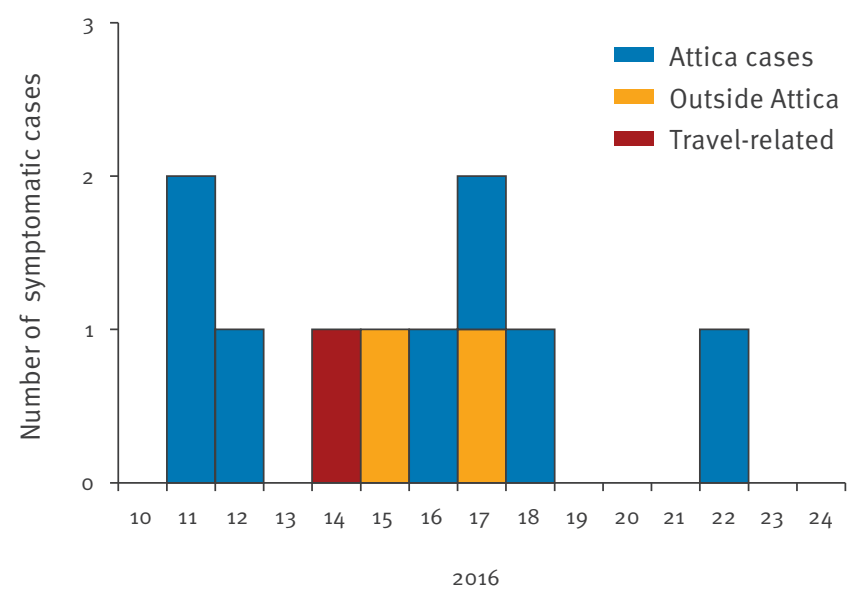

Week of symptom onset

Salmonella enterica susp. enterica with the antigenic type $11: z_{41}: \mathrm{e}, \mathrm{n}, \mathrm{z}_{15}$

\section{Acknowledgements}

We thank our colleagues from the Veterinary Reference Centre for Salmonella in Chalkis for serotyping some of the isolates for confirmation of the antigenic type. We also thank Saara Kotila at ECDC in Stockholm and Francois-Xavier Weill at Pasteur Institute in Paris, for their support.

\section{Conflict of interest}

None declared.

\section{Authors' contributions}

Georgia Mandilara: conception and design of the work and laboratory investigation;

Kleon Karadimas: laboratory investigation;

Kassiani Mellou: conception and design of the work and epidemiological investigation;

Leonidas Georgalis: epidemiological investigation;

Michalis Polemis: PFGE profiles analysis and interpretation;

Theano Georgakopoulou: coordination of the project;

Alkiviades Vatopoulos: coordination of the project.

\section{References}

1. World Health Organization (WHO) / Institut Pasteur. WHO Collaborating Centre for Reference and Research on Salmonella. Antigenic Formulae of the Salmonella serovars. 2007. 9th edition. Paris: WHO Collaborating Centre for Reference and Research on Salmonella. Available from: http:// www.scacm.org/free/Antigenic\%20Formulae $\% 200$ \% 20 the $\% 20$ Salmonella $\% 20$ Serovars $\% 202007 \% 209$ th\%20edition. pdf

2. European Committee on Antimicrobial Susceptibility Testing (EUCAST). [Internet]. Accessed 23 Jun 2016. Växjö: EUCAST. Available from: http://www.eucast.org/

3. Centers for Disease Control and Prevention (CDC). Standard Operating Procedure for PulseNet PFGE of Escherichia coli O157: $\mathrm{H}_{7}$, Escherichia coli non-0157 (STEC), Salmonella serotypes, Shigella sonnei and Shigella flexneri. Atlanta: CDC; Apr 2013. Available from: http://www.cdc.gov/pulsenet/pdf/ ecoli-shigella-salmonella-pfge-protocol-508c.pdf

4. Applied Maths. Bionumerics v6.0.

5. Pogreba-Brown K, Ernst K, Harris RB. Case-case methods for studying enteric diseases: A review and approach for standardization.OA Epidemiology.2014;2(1):7.

6. Gobin M, Launders N, Lane C, Kafatos G, Adak B. National outbreak of Salmonella Java phage type 3 b variant 9 infection using parallel case-control and case-case study designs, United Kingdom, July to October 2010.Euro Surveill. 2011;16(47):20023.PMID: 22152706

7. Krumkamp R, Reintjes R, Dirksen-Fischer M. Case-case study of a Salmonella outbreak: an epidemiologic method to analyse surveillance data.Int J Hyg Environ Health. 2008;211(1-2):163-7. DOI: 10.1016/j.ijheh.2007.02.006 PMID: 17412638

\section{License and copyright}

This is an open-access article distributed under the terms of the Creative Commons Attribution (CC BY 4.0) Licence. You may share and adapt the material, but must give appropriate credit to the source, provide a link to the licence, and indicate if changes were made.

This article is copyright of the authors, 2016. 